\title{
Kommunikativ-pragmatische Störungen bei Alzheimer-Demenz
}

\section{Communicative-Pragmatic Disorders in Alzheimer's Dementia}

Christina Knels

\begin{abstract}
Die Alzheimer-Demenz hat spezifische kommunikativ-pragmatische Defizite, aber auch spezifische Ressourcen, die in der Kommunikation mit Betroffenen genutzt werden können. Die Störung der Pragmatik ist sowohl auf bestehende sprachliche Defizite als auch auf kognitive Defizite zurückzuführen. Bei Beeinträchtigungen in der Verarbeitung nicht wörtlicher Sprache scheinen kognitive Funktionen eine größere Rolle zu spielen als sprachliche.
\end{abstract}

\section{LERNZIEL}

Die Alzheimer-Demenz wird hinsichtlich ihrer sprachlichen und kommunikativ-pragmatischen Beeinträchtigungen und Ressourcen dargestellt. Es wird Wissen über die Ursache der Pragmatikstörung bei leichtgradiger Alzheimer-Demenz und über deren Implikation für die Therapie vermittelt.

\section{Einleitung}

Die Alzheimer-Demenz ist die am häufigsten auftretende Demenzform im höheren Alter. Sie zeichnet sich aus durch einen schleichenden Beginn und ein langsames Fortschreiten von kognitiven Beeinträchtigungen, die in der Konsequenz Probleme in der Bewältigung des Alltags nach sich ziehen. Die beobachtbaren Symptome zeigen sich in den Kernbereichen Kognition, Verhalten und Aktivität.

\section{Kombinierter Schädigungsprozess}

Sprach- bzw. Kommunikationsstörungen sind typisch bei Alzheimer-Demenz und können schon früh im Krankheitsverlauf auftreten [1]. Ursächlich sind dabei 2 Schädigungsprozesse:

- Zum einen liegt eine Beeinträchtigung der Sprachsystematik durch eine Schädigung (Atrophie) der sprachrelevanten Hirnareale zugrunde.

- Zum anderen liegen kognitive Defizite vor, die exekutive (Planungsfunktionen), mnestische (Gedächtnis) und attentionale Beeinträchtigungen (Aufmerksamkeit) beinhalten und ebenfalls Sprache und Kommunikation beeinflussen [2].

\section{Merke \\ Sprach- und Kommunikationsstörungen bei Alzhei- mer-Demenz resultieren aus einer Kombination von aphasischen (sprachsystematischen) und nicht apha- sischen (kognitiven) Symptomen. Diese beeinflussen beide sowohl den Sprach-Output als auch die Kom- munikation bei Alzheimer-Demenz.}

\section{HINTERGRUNDWISSEN}

\section{Kognitive Beeinträchtigungen bei Alzheimer-Demenz}

- Kognition: Frühe Symptome sind Gedächtnisstörungen, visuokonstruktive Defizite und bereits sprachlich-kommunikative Beeinträchtigungen. Weitere Beeinträchtigungen zeigen sich u. a. in Orientierung, exekutiven Funktionen, Aufmerksamkeit und Lernen.

- Verhalten: Im frühen Stadium sind Depressionen, Reizbarkeit, Stimmungsschwankungen und Antriebsverlust zu beobachten. Im Verlauf können aggressives Verhalten, motorische Unruhe, gene-

relles Misstrauen, Wahnphänomene und Sinnestäuschungen auftreten.

- Aktivität: Betroffene ziehen sich früh aus dem sozialen und gesellschaftlichen Leben zurück und Hobbys werden aufgegeben. Im Verlauf sind häusliche Aktivitäten wie das selbstständige Einkaufen bzw. Kochen, der Umgang mit Geld, die persönliche Hygiene und auch die Nahrungsaufnahme nicht mehr ohne Hilfe möglich [1]. 


\section{Sprachlich-kommunikative Symptomatik}

Die Symptomatik bei Alzheimer-Demenz umfasst potenziell alle sprachlichen Ebenen und Modalitäten ( $\triangleright$ Tab. 1). Die sprachlichen Fähigkeiten verschlechtern sich mit Fortschreiten der Erkrankung. Dabei ist allerdings nicht jede sprachliche Ebene zu gleicher Zeit und in gleichem Maße betroffen [3]. Am stärksten beeinträchtigt bzw. schon früh auffällig sind bei Alzheimer-Demenz kommunikativ-pragmatische und lexikalisch-semantische Fähigkeiten, gefolgt von syntaktischen Leistungen. Am wenigsten häufig sind phonologische Auffälligkeiten zu beobachten [3].

\section{Merke}

Bei Alzheimer-Demenz sind kommunikativ-pragmatische sowie lexikalisch-semantische Fähigkeiten bereits im frühen Stadium beeinträchtigt und im Verlauf am schwersten betroffen. Morphosyntaktische und phonologische Auffälligkeiten manifestieren sich erst im Verlauf der Erkrankung.

\section{Last-in-first-out-Hypothese}

Der Verlust sprachlicher Funktionen geht hierarchisch vonstatten. Dabei sind die Aspekte der Sprache, die später erworben wurden, zuerst betroffen (sog. Last-
in-first-out-Hypothese) [4]. Das Erwerbsalter spielt beispielsweise bei der Störungsanfälligkeit von Lexemen eine wichtige Rolle: Betroffene produzieren und verstehen insbesondere Wörter, die früher erworben wurden, im Gegensatz zu Wörtern, die eher später erworben wurden $[5,6]$.

\section{Störung der Kommunikation bei Alzheimer-Demenz}

\section{Allgemeines Kommunikationsverhalten}

Es konnte nachgewiesen werden, dass bereits Kommunikationsstörungen bei Alzheimer-Demenz vorliegen, während sprachstrukturelle Fähigkeiten noch weitgehend intakt sind [7]. In der Kommunikation zeigen Betroffene im frühen Stadium schon Schwierigkeiten in der aktiven Aufrechterhaltung des Themas bzw. dem Verfolgen der propositionellen Entwicklung des Themas einer Konversation (sog. roter Faden). Auch bestehen Schwierigkeiten in der erfolgreichen Verfolgung von Themenwechseln und eine generelle Problematik, sich in der Kommunikation an den Wissensstand bzw. die Perspektive des Gesprächspartners anzupassen $[8,9]$. Ein oft in der Praxis beobachtetes Phänomen in Narrationen von Alzheimer-Demenz Betroffener ist beispielsweise die definite Ersterwähnung einer 3. Per-

Tab. 1 Störungen der Sprache und der Kommunikation bei Alzheimer-Demenz (Quelle: Knels C. Sprache und Ernährung bei Demenz. Stuttgart: Thieme; 2018).

\begin{tabular}{|c|c|c|c|}
\hline Sprachebene & Frühes Stadium & Mittleres Stadium & Spätes Stadium \\
\hline $\begin{array}{l}\text { Pragmatik und } \\
\text { Kommunikation }\end{array}$ & $\begin{array}{l}\text { Diskursstörungen (Verlust des „roten } \\
\text { Fadens“ bzw. Themaverlust, gestörte } \\
\text { Adaption an Vorwissen des Ge- } \\
\text { sprächspartners), weitschweifig } \\
\text { Ressource: Aufmerksamkeit weit- } \\
\text { gehend intakt }\end{array}$ & $\begin{array}{l}\text { Kohärenzdefizite, verminderter } \\
\text { Informationsgehalt (Verlust von } \\
\text { Relevanz und Qualität der Inhalte) } \\
\text { Ressource: nonverbale Kommunika- } \\
\text { tion }\end{array}$ & $\begin{array}{l}\text { kaum erfolgreiche verbale Kommuni- } \\
\text { kation mehr möglich, Intrusionen, } \\
\text { Mutismus } \\
\text { Ressource: Kommunikation auf Be- } \\
\text { ziehungsebene über Mimik, Gestik } \\
\text { bzw. Augenkontakt }\end{array}$ \\
\hline $\begin{array}{l}\text { Semantik und } \\
\text { Lexikon bzw. } \\
\text { Wortfindung }\end{array}$ & $\begin{array}{l}\text { Wortfindungs- und Benennstörun- } \\
\text { gen, Wortwahl weniger präzise, } \\
\text { Floskeln, Wiederholungen }\end{array}$ & $\begin{array}{l}\text { semantische Paraphasien mit zu- } \\
\text { nächst nur geringer Abweichung vom } \\
\text { Zielwort, deutliche Wortfindungs- } \\
\text { störungen auch bei hochfrequenten } \\
\text { Wörtern, häufige Wiederholungen, } \\
\text { Verwendung von Prototypen }\end{array}$ & $\begin{array}{l}\text { Wiederholung bedeutungsloser } \\
\text { Wörter und/oder Geräusche, sehr } \\
\text { viele semantische Paraphasien, } \\
\text { semantischer Jargon, inhaltsleere } \\
\text { Restsprache }\end{array}$ \\
\hline $\begin{array}{l}\text { (Sprach-) } \\
\text { Verständnis }\end{array}$ & $\begin{array}{l}\text { Schwierigkeiten im Verständnis } \\
\text { komplexer Sätze } \\
\text { Ressource: Verständnis einfacher } \\
\text { Sätze und Einzelwortverständnis }\end{array}$ & $\begin{array}{l}\text { Störungen im Hörverstehen auf } \\
\text { Wortebene } \\
\text { Ressource: Verstehen nonverbaler } \\
\text { Botschaften }\end{array}$ & $\begin{array}{l}\text { Sprachverständnis auf Inhaltsebene } \\
\text { massiv beeinträchtigt } \\
\text { Ressource: Verstehen auf Bezie- } \\
\text { hungsebene, Interpretieren emotio- } \\
\text { naler Zustände über Mimik, Gestik, } \\
\text { Augenkontakt, Prosodie bzw. Stimm- } \\
\text { klang }\end{array}$ \\
\hline Syntax & $\begin{array}{l}\text { Vereinfachungsstrategien in der } \\
\text { Satzbildung } \\
\text { Ressource: grammatikalisch korrek- } \\
\text { te Sätze }\end{array}$ & $\begin{array}{l}\text { häufig Satzfragmente und Satzver- } \\
\text { schränkungen, kaum Nebensätze, } \\
\text { morphosyntaktische Fehler }\end{array}$ & $\begin{array}{l}\text { ausschließlich Satzfragmente und } \\
\text { Satzverschränkungen }\end{array}$ \\
\hline $\begin{array}{l}\text { Phonologie und } \\
\text { Artikulation }\end{array}$ & $\begin{array}{l}\text { Ressource: Lautbildung bzw. Artiku- } \\
\text { lation meist unauffällig }\end{array}$ & phonematische Paraphasien & $\begin{array}{l}\text { sehr viele phonematische Parapha- } \\
\text { sien, verwaschene Aussprache }\end{array}$ \\
\hline
\end{tabular}


son mit Namen (z.B. „... und die Elisabeth war auch mit dabei."), obwohl im Kommunikationskontext klar sein sollte, dass der Hörer nicht wissen kann, wer Elisabeth ist. Einen kommunikativen Perspektivwechsel vorzunehmen, involviert Fähigkeiten der Theory of Mind.

\section{HINTERGRUNDWISSEN \\ Theory of Mind}

Theory of Mind ist die Fähigkeit, sich in andere Menschen „hineinzuversetzen“, um ihre Handlungen, Absichten und Gefühle einschätzen zu können. Durch Theory of Mind wird der Mensch befähigt, anderen Personen geistige Prozesse zuzuschreiben, z. B. deren Überzeugungen, Absichten, Wünsche oder Gefühle. Dabei wird in die kognitive und die affektive Theory of Mind unterteilt:

- kognitive Theory of Mind: Fähigkeit, anderen Menschen bestimmte Annahmen oder Überzeugungen zuzuordnen,

- affektive Theory of Mind: Fähigkeit, Personen bestimmte Gefühlszustände zuzuschreiben.

Es besteht ein signifikanter Zusammenhang zwischen Theory-of-Mind-Fähigkeiten und pragmatischen Leistungen [10]. Von Alzheimer-Demenz Betroffene im frühen Stadium zeigen hinsichtlich komplexer kognitiver Theory of Mind klare Beeinträchtigungen, wohingegen sie basale kognitive Theory-of-Mind-Aufgaben noch gut bewältigen können. Bei Aufgaben zur affektiven Theory of Mind schneiden Betroffene im frühen Stadium meist nicht anders ab als gesunde Kontrollprobanden [11].

Im Verlauf manifestieren sich immer deutlichere Kohärenzdefizite, Konfabulationen und ein zunehmend verminderter Informationsgehalt der Äußerungen. Im späten Stadium ist eine erfolgreiche verbale Kommunikation nahezu unmöglich. Im schlimmsten Fall interagieren Betroffene kaum mehr in irgendeiner Weise mit der Außenwelt [1].

\section{Basisleistungen der Kommunikation}

Um erfolgreich kommunizieren zu können, sind nicht allein sprachliche Produktions- und Rezeptionsleistungen notwendig: Der Aufmerksamkeitsfokus des Hörers muss auf dem Sprecher liegen, damit der Hörer die Botschaft überhaupt wahrnehmen und dekodieren kann. Die verstandene Botschaft sollte anschließend behalten werden (Gedächtnis), damit sich Themen im Dialog propositionell überhaupt entwickeln können (Was wurde bereits gesagt? Was muss noch gesagt werden?). Bei Alzheimer-Demenz sind die Basisfunk-

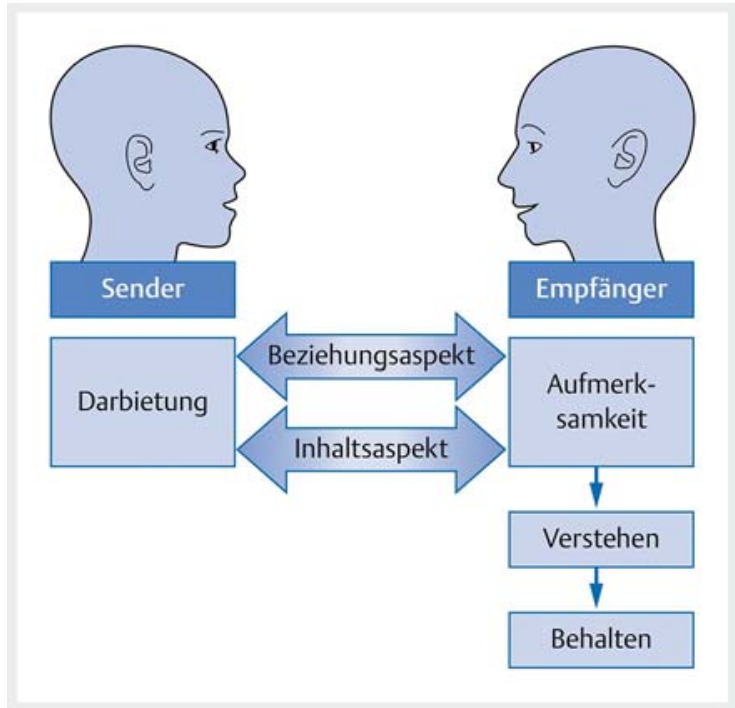

Abb. 1 Kombiniertes Kommunikationsmodell. Quelle: Knels C. Sprache und Ernährung bei Demenz. Stuttgart: Thieme; 2018

tionen, die für eine grundlegende Aufrechterhaltung einer Kommunikation wichtig sind, schlussendlich alle beeinträchtigt (Produktion, Aufmerksamkeit, Verstehen, Behalten). Innerhalb dieser Bereiche bleiben dennoch Ressourcen erhalten, die es in der Kommunikation zu nutzen bzw. in der Therapie zu fördern gilt ( Abb. 1) $[1,12]$.

\section{Kommunikative Ressourcen}

Trotz sprachlicher und kognitiver Defizite sind bei Alzheimer-Demenz kommunikative Ressourcen vorhanden (s. > Tab. 1). Aufmerksamkeitsleistungen sind bei Alzheimer-Demenz zwar insgesamt beeinträchtigt, das Fokussieren der Aufmerksamkeit gelingt jedoch bei leichter Alzheimer-Demenz noch relativ gut. Hingegen bereitet das Verschieben oder Teilen der Aufmerksamkeit bereits Schwierigkeiten [12]. Gedächtnisinhalte, die weit in der Vergangenheit liegen (Altgedächtnis), können bei Alzheimer-Demenz besser abgerufen werden als Inhalte, die erst kurze Zeit zurückliegen $[12,13]$. Biografieorientierte Themen aus Kindheit bzw. Jugend bieten sich daher zur Kommunikationserleichterung an. Ebenso können sehr bedeutsame Lebensereignisse und emotional behaftete autobiografische Erlebnisse besser erinnert werden, auch wenn sie noch nicht so lange zurückliegen (Emotional Memory Effect) $[12,14]$. Insgesamt zeigt sich die Sachebene der Kommunikation beeinträchtigt, wohingegen die Kommunikation auf Beziehungsebene auch bei fortgeschrittener Demenz noch relativ gut erhalten ist. Dies betrifft insbesondere die Produktion und das Verständnis paraverbaler (z. B. Stimmklang, Sprachmelodie, Betonung, Lautstärke) und nonverbaler Kommunikationsmittel (Gestik, Mimik, Haltung, Berührung) [1]. 


\section{Merke}

Neben kommunikativen Defiziten sind auch kommunikative Ressourcen bei Alzheimer-Demenz zu finden, z. B. Altgedächtnis, Emotionen und Kommunikation auf Beziehungsebene. Diese sollten in der Kommunikation mit Betroffenen gezielt genutzt und gefördert werden.

\section{Störung der nicht wörtlichen Sprache bei Alzheimer-Demenz}

Hinsichtlich Störungen im Verständnis nicht wörtlicher Sprache bei leichtgradiger Alzheimer-Demenz zeigt sich die Studienlage teilweise heterogen. Das ist insbesondere auf die Verwendung unterschiedlicher Testverfahren und -modalitäten zurückzuführen $[15,16]$. Im Folgenden werden die Leistungen bei AlzheimerDemenz bezüglich nicht wörtlicher Sprache exemplarisch anhand der Verarbeitung von Metaphern, Idiomen und Sarkasmus dargestellt.

\section{Metaphern}

Konventionelle Metaphern, d. h. Metaphern, die bereits lexikalisiert sind (z. B. „Rabeneltern“, eine „spitze Bemerkung“), zeigen sich bei leichter Alzheimer-Demenz eher unbeeinträchtigt. Unkonventionelle Metaphern sind im alltäglichen Sprachgebrauch nicht etabliert, daher ist das Verständnis der Metapher nur unter Zuhilfenahme des Kontextes möglich. Das Verständnis unkonventioneller Metaphern ist bereits bei leichter Alzheimer-Demenz beeinträchtigt. Interessanterweise zeigte sich nicht vorrangig das Sprachverständnis als ursächlich für das schlechte Verständnis unkonventioneller Metaphern, sondern die exekutiven Funktionen [17].

\section{Idiome}

Idiome (Redewendungen) haben oftmals einen bildhaften Charakter (z. B. „einen grünen Daumen haben“) und unterscheiden sich von Metaphern dadurch, dass mehrere Wörter fest zu einer Einheit verbunden sind. Dabei lässt sich die Gesamtbedeutung nicht unmittelbar aus der Bedeutung der einzelnen Elemente erschließen. Die Verarbeitung von Idiomen ist im frühen Stadium der Alzheimer-Demenz bereits beeinträchtigt. Dabei wird häufig die wörtliche Bedeutung des Idioms statt der übertragenen Bedeutung verarbeitet $[15,16$, 18]. Ursächlich für die beeinträchtigte Verarbeitung von Idiomen ist die Kombination von semantischen und exekutiven Störungen [15]. Die übertragene Bedeutung der Idiome ist jedoch bei leichter AlzheimerDemenz nicht unbedingt „verloren“: Ausschlaggebend für die Problematik ist vielmehr die beeinträchtigte Fähigkeit, die wörtliche Bedeutung zugunsten der übertragenen Bedeutung zu hemmen. Als primäre Ursache wird daher eher die exekutive Störung ausgemacht [18].

\section{Sarkasmus}

Sarkasmus und Ironie sind sprachliche Mittel, bei denen der Sprecher Wörter verwendet, die sich eigentlich auf das Gegenteil des wörtlich Gesagten beziehen, meist, um indirekt einen Missstand zu verdeutlichen (z. B. „Schönes Wetter heute!“ bei Gewitter). Sarkasmus ist eine Form von Ironie, allerdings mit höhnischspottender oder verletzender Intention. Um Sarkasmus zu verstehen, muss der Hörer nicht nur erkennen, dass der Sprecher nicht gemeint hat, was er sagt, sondern auch, dass das Gesagte nicht wörtlich genommen werden darf. Das erfolgreiche Verarbeiten von sarkastischen Aussagen involviert Theory-of-Mind-Prozesse [16].

Die Verarbeitung von sarkastischen Aussagen ist bei von Alzheimer-Demenz Betroffenen bereits früh beeinträchtigt, allerdings nur bei Aufgaben, in denen Sarkasmus rein aus dem Kontext heraus erfasst werden muss (z. B. Lesen und Beurteilen von kurzen Szenarien). Dabei nehmen Betroffene sarkastische Aussagen sehr oft wörtlich [19]. Werden die Aufgaben allerdings so gestellt, dass neben dem reinen Kontext auch paraverbale (z. B. Tonfall) bzw. nonverbale Hinweise (z.B. Mimik) gegeben werden (z. B. Sehen und Beurteilen von Szenarien in Videosequenzen), unterscheidet sich die Leistung nicht mehr von Gesunden [20]. Betroffene profitieren also in der Verarbeitung von paraverbalen und nonverbalen Aspekten, die die Beziehungsebene in der Kommunikation bedienen.

FAZIT

Kommunikativ-pragmatischen Beeinträchtigungen bei leichtgradiger Alzheimer-Demenz liegt ein Ursachenkomplex zugrunde, bestehend aus sprachlichen (insbesondere lexikalisch-semantischen) und kognitiven Defiziten. Insbesondere in der Verarbeitung nicht wörtlicher Sprache scheinen kognitive Leistungseinbußen eine größere Rolle zu spielen als sprachliche. Für die therapeutische Herangehensweise bedeutet dies, dass eine Therapie von Sprachfunktionen dabei nicht zielführend ist. Stattdessen kommt dem Dialog innerhalb einer kommunikations- und ressourcenorientierten Therapie bei Alzheimer-Demenz eine besondere Bedeutung zu. Erhaltene Kanäle der Mitteilung sind auf der Beziehungsebene verortet: Gestik, Mimik, Stimmklang, Körperhaltung und Berührung sind entscheidend, um Emotionen zu vermitteln und die Kommunikation bei Alzheimer-Demenz zu erhalten und zu fördern. 


\section{Schlüsselwörter}

Alzheimer-Demenz, Kommunikation, Pragmatik, nicht wörtliche Sprache

\section{Interessenkonflikt}

Die Autorinnen/Autoren geben an, dass kein Interessenkonflikt besteht.

\section{Autorinnen/Autoren}

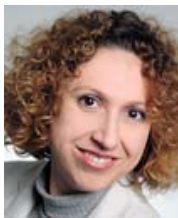

\section{Christina Knels}

Im Anschluss an das Studium der klinischen Linguistik Tätigkeit als Sprachtherapeutin im Bereich Neurologie/ Geriatrie. Nach der Promotion Arbeit als wissenschaftliche Mitarbeiterin an der LMU München, und an der Hochschule Fresenius Hamburg. Seit 2014 Professorin für Neurowissenschaften und Neurolinguistik an der Medical School Hamburg.

Institut

Medical School, Hamburg

Korrespondenzadresse

Christina Knels

Fakultät Gesundheitswissenschaften

MSH Medical School Hamburg

University of Applied Sciences and Medical University

Am Kaiserkai

20457 Hamburs

E-Mail: christina.knels@medicalschool-hamburg.de

Literatur

[1] Knels C. Sprache und Ernährung bei Demenz. Stuttgart: Thieme; 2018

[2] Gutzmann H, Brauer T. Sprache bei Demenz. Idstein: Schulz-Kirchner; 2007

[3] Boschi V, Catricalà E, Consonni M et al. Connected speech in neurodegenerative language disorders: a review. Front Psychol 2017; 8: 269. doi: 10.3389/fpsyg.2017.00269

[4] Cummings L. Pragmatic disorders. Heidelberg: Springer; 2014. doi: 10.1007/978-94-007-7954-9

[5] Sailor KM, Zimmerman ME, Sanders AE. Differential impacts of age of acquisition on letter and semantic fluency in Alzheimer's disease patients and healthy older adults. Q J Exp Psychol 2011; 64: 2383-2391. doi: 10.1080/ 17470218.2011 .596660
6] Cuetos F, Herrera E, Ellis AW. Impaired word recognition in Alzheimer's disease: the role of age of acquisition. Neuropsychologia 2010; 48: 3329-3334

[7] Bayles KA, Tomoeda CK, Trosset MW. Relation of linguistic communication abilities of Alzheimer's patients to stage of disease. Brain Language 1992; 42: 454-472

[8] Mentis M, Briggs-Whittaker J, Gramigna GD. Discourse topic management in senile dementia of the Alzheimer's type. J Speech Hear Res 1995; 38: 1054-1066

[9] Romero B. Sprachverhaltensstörungen bei Morbus Alzheimer. In: Weis S, Weber G, Hrsg. Handbuch Morbus Alzheimer. Weinheim: Psychologie Verlags Union; 1997: 921-973

[10] Cuerva AG, Sabe L, Kuzis G et al. Theory of mind and pragmatic abilities in dementia. Neuropsychiatry Neuropsychol Behav Neurol 2001; 14: 153-158

[11] Poletti M, Enrici I, Adenzato M. Cognitive and affective theory of mind in neurodegenerative diseases: neuropsychological, neuroanatomical and neurochemical levels. Neurosci Biobehav Rev 2012; 36: 2147-2164. doi: 10.1016/ j.neubiorev.2012.07.004

[12] Haberstroh J, Pantel J. Kommunikation bei Demenz - TANDEM, Trainingsmanual. Berlin: Springer; 2011

[13] Sartori G, Snitz BE, Sorcinelli L et al. Remote memory in advanced Alzheimer's disease. Arch Clin Neuropsychol 2004; 19: 779-789

[14] Kalenzaga S, Piolino P, Claris D. The emotional memory effect in Alzheimer's disease: emotional words enhance recollective experience similarly in patients and control participants. Cogn Emot 2015; 29: 342-350. doi: 10.1080/ 02699931.2014 .907127

[15] Rassiga C, Lucchelli F, Crippa F et al. Ambiguous idiom comprehension in Alzheimer's disease. J Clin Exp Neuropsychol 2009; 31: 402-411

[16] Rapp A, Wild B. Nonliteral language in Alzheimer dementia: a review. J Int Neuropsychol Soc 2011; 17: 207-218. doi: $10.1017 /$ S1355617710001682

[17] Amanzio M, Geminiani G, Leotta D et al. Metaphor comprehension in Alzheimer's disease: novelty matters. Brain Lang 2008; 107: 1-10

[18] Papagno C, Lucchelli F, Muggia S et al. Idiom comprehension in Alzheimer's disease: the role of the central executive. Brain 2003: 126: 2419-2430

[19] Maki Y, Yamaguchi T, Koeda T et al. Communicative competence in Alzheimer's disease: metaphor and sarcasm comprehension. Am J Alzheimers Dis Other Demen 2013; 28: 69-74. doi: 10.1177/1533317512467677

[20] Rankin KP, Salazar A, Gorno-Tempini ML et al. Detecting sarcasm from paralinguistic cues: anatomic and cognitive correlates in neurodegenerative disease. Neuroimage 2009; 47: 2005-2015

Bibliografie

DOI https://doi.org/10.1055/a-1043-7822 Online-Publikation: 21.4.2020 | Sprache · Stimme · Gehör 2020; 44: 90-94

(c) Georg Thieme Verlag KG Stuttgart · New York ISSN 0342-0477 